\title{
Single Crystal Diamond Schottky Photodiode
}

\author{
Claudio Verona \\ Dip. di Ing. Meccanica, Università di Roma "Tor Vergata", Roma \\ Italy
}

\section{Introduction}

Thanks to its extreme optical and electronic properties, diamond appears to be a promising semiconducting material for photon detection. Its wide band-gap, $5.5 \mathrm{eV}$, results in a very low leakage current and its electronic properties as high carrier mobility allow fast time response (J. E. Field, 1979). Besides, it has a large breakdown electric field $(\sim 10 \mathrm{~V} / \mu \mathrm{m})$, a low dielectric constant (i.e. low capacitance), chemical inertness and low intrinsic carrier density, which makes cooling for noise reduction unnecessary (J.Prins, 1997). Its extreme radiation hardness is well known and another interesting feature, again related to the wide band-gap, is its selective sensitivity to radiation with wavelengths shorter than $225 \mathrm{~nm}$ (visible-blind detectors) (J.F. Hochedez et al., 2002). Several attempts have been made to build up UV detectors from natural or synthetic diamonds grown by Chemical Vapour Deposition (CVD). A detector often reported in literature is the photoresistor (A. Balducci e al., 2005; T. Teraji et al., 2004) having a planar structure and consisting of a photoconductive diamond film with metal electrodes placed on the top surface. It can operate only with external voltage applied and the signal is affected from secondary electron emission, which is known to strongly affect the detection properties in the UV and EUV spectral regions. A different geometry reported is a polycrystalline sandwiched photodiode structure (V.I. Polyakov et al., 1998, L. Thaiyotin et al., 2002) with a contact on the diamond growth surface and a backside contact on the silicon substrate. However, the CVD diamond performance is limited in this case by the polycrystalline structure due to defect states in the band gap introduced by the grain boundaries (R. D. McKeag\&R. B. Jackman, 1998, L. Barberini, 2001), which affects the photoelectric properties and alters the detection characteristics. On the other hand, detector grade natural diamonds are extremely rare and expensive, while high pressure high temperature (HPHT) diamonds have their performance strongly worsened by defects and impurities (E. Pace et a., 2000). A great effort is therefore being devoted to produce device-grade Single Crystal Diamond films (SCD) by homoepitaxial CVD growth on low-cost diamond substrates (S. Almaviva et al., 2009, 2010a). A few years ago, at the University of Rome "Tor Vergata" laboratories, CVD single crystal diamond films were used to obtain a new class of detectors with a layered structure. Thanks to the combination of boron doped and intrinsic single crystal diamond films, together with the possibility to easily build Schottky junctions on intrinsic diamond by thermal evaporation of the metal contacts, it has been possible, by using simple multilayered a p-type/nominally intrinsic diamond/metal layered structures, to obtain high quality and highly reproducible devices which can be effectively used for detection (UV and X-rays) photons. 
In this chapter, the fabrication and characterization of two Schottky photodiodes, based on p-type/intrinsic diamond/metal (PIM) Schottky junction in two different operative configurations is reported (S. Almaviva et al., 2010b) . One detector has been designed in transverse configuration with a semitransparent metallic contact evaporated on the diamond surface, while the second one operates in a planar configuration with interdigitated fingers on the diamond surface. One set of fingers is made of aluminium and the second one is made of p-type diamond.

\section{CVD single crystal diamond films production (detector grade)}

The reactor for Microwave Plasma Enhanced Chemical Vapour Deposition (MPECVD) diamond growth, used at the Mechanical Engineering Department of the University of Rome "Tor Vergata" laboratories is illustrated in Fig.1. This apparatus is used for the deposition of diamond films in the present work. This deposition chamber was built by developing the necessary parameters to obtain films of diamond having the best physical characteristics, together with a high degree of reproducibility results.

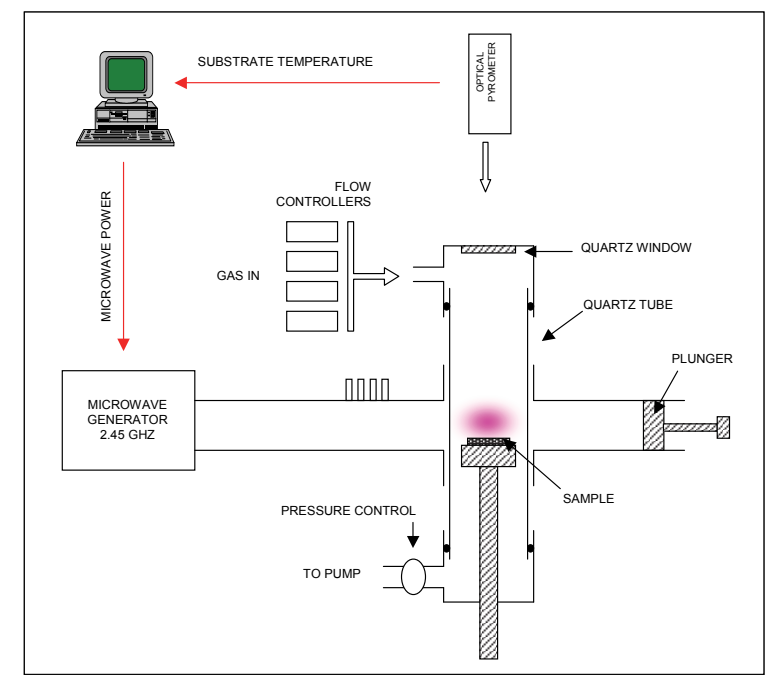

Fig. 1. General scheme of the apparatus for CVD diamond films deposition

The apparatus for CVD diamond deposition is composed by a tubular microwave CVD reactor and the growth chamber consists essentially in a quartz tube into which the precursory gases flow, controlled by four flowmeters, (a mixture $100 \mathrm{H}_{2} / 1 \mathrm{CH}_{4} \mathrm{sccm}$ for the intrinsic growth and $90 \div 70 \mathrm{H}_{2} / 2 \mathrm{CH}_{4} / 10 \div 30 \mathrm{H}_{2}-\mathrm{B}_{2} \mathrm{H}_{6} \mathrm{sccm}$ for the doped one) at a stationary fixed pressure (typically $120 \div 130 \mathrm{mBar}$ ).

The tube is put across a waveguide connected to a commercial $2.45 \mathrm{GHz}$ MW generator (Sairem ${ }^{\circledR}$ magnetron, $2 \mathrm{~kW}$ maximum MW power) and the typical microwave power for both SCD growths is $500 \div 550 \mathrm{~W}$. The quartz tube acts then as a resonant cavity for the microwaves and a stable plasma burns at the centre of the tube, coupling to the sample holder, that is properly connected to a water cooling system, and thus to the diamond substrate on which the homoepitaxial deposition process takes place. The position of the 
plasma can be modified through a "plunger" (a sliding short circuit) that terminates the waveguide and its coupling with the substrate can be optimized by means of a 3-stub tuner put between the MW generator and the quartz tube. The temperature of the growing film is monitored in real-time through an infrared optical pyrometer and can be maintained constant during the whole CVD process thanks to a proper software which follows the growth and varies properly the MW power supplied by the generator. In this type of reactor, microwaves give energy to the electrons of the plasma, which, at his time, give energy to the gas through molecular collisions, heating it. The chemical and physical reactions, which follow this heating, allow the formation of carbon that is deposited on diamond substrate. The chemistry of the diamond deposition is described in the next paragraph. It's worth underline that diamond growth is obtained only if growth parameters (gas composition, pressure, plasma temperature, microwave power density, etc) are chosen in the appropriate way. In particular, the grow rate of diamond with this method vary from $0.2 \mu \mathrm{m} / \mathrm{h}$ to $10 \mu \mathrm{m} / \mathrm{h}$, depending on growth parameters and chamber geometry.

\section{P-type/ intrinsic diamond/ metal (PIM) Schottky photodiode}

Schottky photodiodes are based on synthetic SCD films produced in our laboratories by using MPECVD technique previously explained.

The nominally intrinsic diamond is deposited by using a completely separated apparatus in order to avoid any boron contamination. In fact, the presence of impurities in the intrinsic SCD active layer would determine a drastic worsening of the resulting performances, in terms of increasing of the dark current, temporal instability, memory effects, priming, slow response times, worsening of the spectroscopic performances, etc. All CVD films were principally characterized by X-ray diffraction, cathodoluminescence and Scanning Electron Microscopy, confirming the single crystal homoepitaxial deposition and the good crystal quality of the grown samples.

The single crystal p-type diamond was grown keeping constant the microwave power, while the intrinsic layer was grown keeping constant the temperature. The typical growth conditions are reported in Table 1.

\begin{tabular}{|c|c|c|}
\hline \hline Typical growth parameters & Intrinsic diamond & p-type diamond \\
\hline Substrate & \multicolumn{2}{|c|}{$(100) \mathrm{HPHT}$ type Ib } \\
\hline Plasma composition & $\mathrm{CH}_{4}-\mathrm{H}_{2}$ & $\mathrm{CH}_{4}-\mathrm{H}_{2}-\mathrm{B}_{2} \mathrm{H}_{6}$ \\
\hline Gas flow rate & $1-100 \mathrm{sccm}$ & $2-100-10 \div 30 \mathrm{sccm}$ \\
\hline Microwave power & $500 \div 600 \mathrm{~W}$ & $\sim 500 \mathrm{~W}$ \\
\hline Temperature & $720^{\circ} \mathrm{C}$ & $450{ }^{\circ} \mathrm{C} \mathrm{--->650} 0^{\circ} \mathrm{C}$ \\
\hline Pressure & $120 \mathrm{mbar}$ & $150 \mathrm{mbar}$ \\
\hline Thickness rate & $1 \mu \mathrm{m} / \mathrm{h}$ & $2 \mu \mathrm{m} / \mathrm{h}$ \\
\hline
\end{tabular}

Table 1. Typical growth parameters

The purity of $\mathrm{CH} 4$ and $\mathrm{H} 2$ gases were $99.9995 \%$ and $99.9999 \%$ respectively. Boron doping was performed by adding dyborane-hydrogen gas mix (100 ppm $\mathrm{B}_{2} \mathrm{H}_{6}$ in hydrogen) to the source gases. In the following section, the fabrication process of both photodiodes with different structures is reported. 


\subsection{Transverse configuration}

The diamond photodiode in transverse configuration consist of a multilayered structure obtained by a two step deposition process. A conductive boron doped diamond homoepitaxial layer (see Fig.2 (b)), used as a backing contact, is deposited, at first, by Microwave Plasma Enhanced Chemical Vapour Deposition (MWPECVD) on a commercial low-cost synthetic High Temperature High Pressure (HPHT) $<100>$ type Ib single crystal diamond (SCD) substrate, $4 \times 4 \times 0.5 \mathrm{~mm}^{3}$ in size and approximately $400 \mu \mathrm{m}$ thick. The boron concentration was estimated by fitting the Resistivity - Temperature curves, obtaining values in the range $10^{20} \mathrm{~cm}^{-3}$. After that a nominally intrinsic diamond layer, which operates as the detecting region, is homoepitaxially grown on the doped one (see Fig.2 (c)). Its thickness can vary from a few microns up to more than 200 microns. Due to the small penetration depth of the UV radiation in the 10-200 nm spectral range (D. Palik., 1991), the detecting region of diamond has a thickness of approximately $2 \mu \mathrm{m}$. In the case of the soft Xray detection, a higher thickness can be chosen (about $30 \mu \mathrm{m}$ ). The nominally intrinsic diamond is deposited by using a completely separated apparatus in order to avoid any boron contamination.

After the growth, each diamond layer has been oxidized by isothermal annealing at $500{ }^{\circ} \mathrm{C}$, for $1 \mathrm{~h}$ in air, in order to remove the $\mathrm{H}_{2}$ surface conductive layer. Finally, a circular metal electrode, about $3 \mathrm{~mm}$ diameter is deposited on the diamond surface by thermal evaporation and annealed silver paint was utilized in order to provide an almost ohmic contact to the B-doped layer (see Fig.2 (d)).
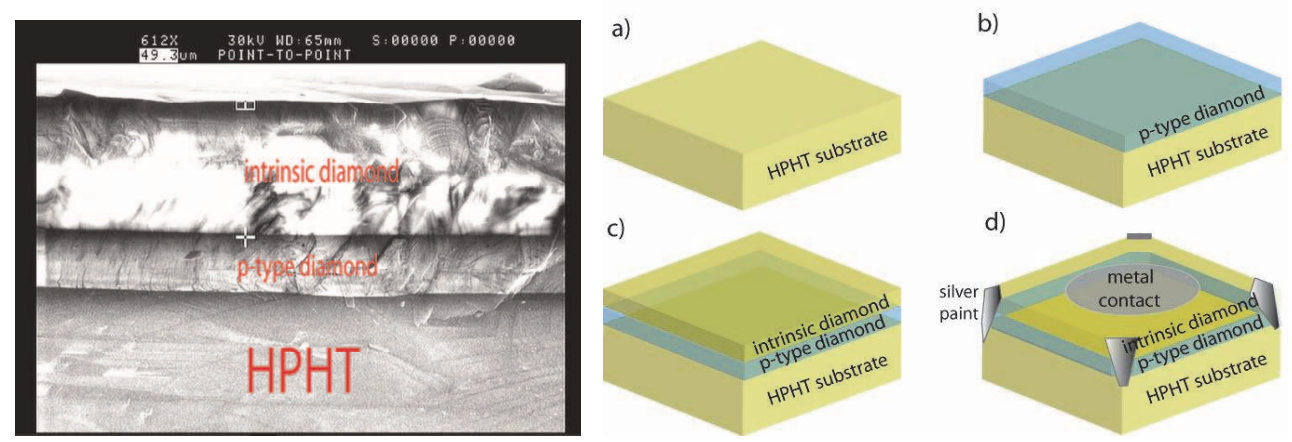

Fig. 2. Diamond deposition process of the PIM detector

Such a structure acts as a p-type/ intrinsic/ metal (PIM) Schottky Barrier Diode: the metal creates a Schottky junction with the intrinsic diamond, which acts as a drift-layer, and the SCD p-type layer, holes injector, determines the unipolarity of the device under direct polarization. The device, therefore, operates in typically reverse biased mode, with a negative voltage on the boron doped contact and the metal top contact grounded.

\subsection{Planar configuration}

In this section, it is report on the fabrication process of extreme ultraviolet (EUV) photovoltaic single crystal diamond Schottky diodes based on metal / intrinsic / p-type diamond junction developed at the University of Rome "Tor Vergata" operates in planar configuration with an interdigitated contact structure on the growth surface of the intrinsic 
diamond layer. One set of fingers is made of aluminium and the second one is made of $\mathrm{p}^{+-}$ type diamond. Two steps of a standard photolithographic technique are used for the fabrication process (see Fig.3) for the diamond detector with interdigitated finger electrodes (IDT-PIM in the following). First, an intrinsic diamond layer is homoepitaxially grown by MWPECVD on a commercial HPHT single crystal diamond substrate. As previously mentioned, annealing in air is employed in order to remove the surface conductive layer of the as-grown diamond film.

After the annealing process, p-type diamond interdigitated fingers are selectively grown on the top of the intrinsic diamond layer by using a patterned $\mathrm{Cr}$ plasma-resistant coplanar mask (Fig.3 (a)-(d)). After removal of the chromium mask by wet etching, the interdigitated $\mathrm{Al}$ electrode is fabricated by using a second mask which is aligned to the pattern previously obtained.

The Al fingers are patterned by standard lift-off photo-lithographic technique and by thermal evaporation on the CVD intrinsic diamond surface (Fig.3 (e)-(g)). The width and the gap between two fingers are both $20 \mu \mathrm{m}$. A SEM image of the IDT-PIM device in Fig.3. The IDT-PIM is simply mounted in an sample holder for UV measurements. In this case, the measured photocurrent of IDT-PIM detector can contain both photoemission current and photoconductive current. The photoemission contribution contains electron emission arising from $\mathrm{Al}$ fingers and from p-type diamond exposed to the UV irradiation.

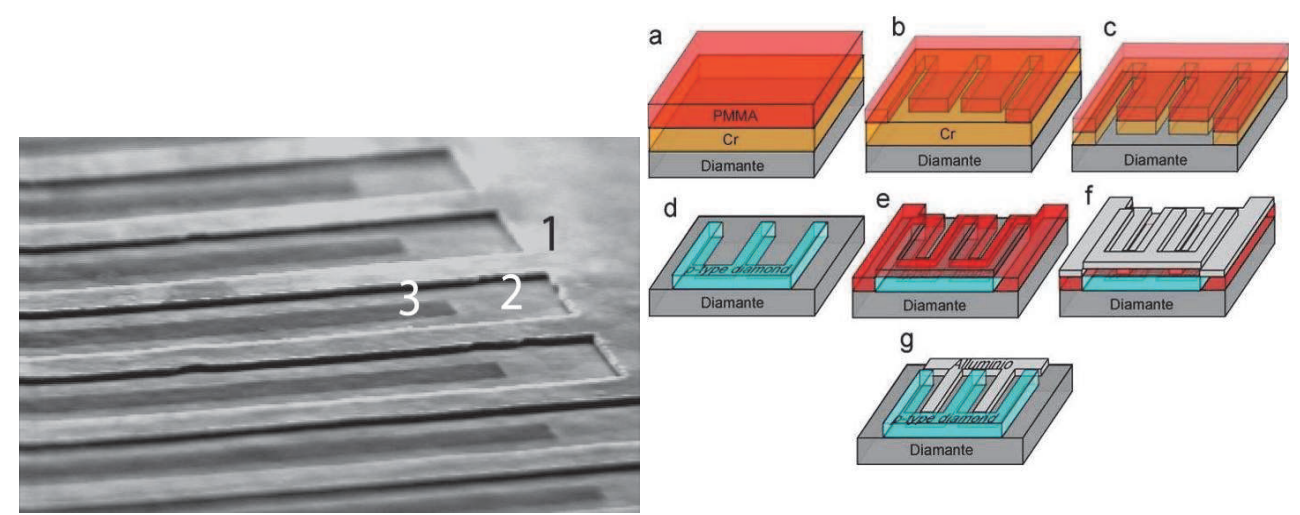

Fig. 3. Fabrication process of the IDT-PIM photodetector and SEM image of the IDT-PIM device: (1) p-type diamond, (2) intrinsic diamond and (3) Al contact.

\section{Electrical characterization}

The investigation of the physical principle of operation of such photodiode through the study of their electrical detection properties is reported in this paragraph. The physics of the devices is clearly based on the existence of a Schottky barrier, as demonstrated by the fact that the detectors are able to operate even with no external bias applied. The aim of the electrical characterization of the devices is, therefore, to analyze the Schottky barrier parameters, measured using the I-V curves from which the barrier height can be extracted. Moreover, the study of metal/p-type diamond contacts to obtain a good ohmic contact is also reported. 


\subsection{Ohmic contact on p-type diamond}

High quality ohmic contacts are one of the moswt frequently encountered problems in the development of the right material for electronic devices applications. The properties of such electrical contacts directly contribute to the active devices performance. In general the requirements for ohmic contacts can be summarized as follows (M. Werner, 2003):

- low contact resistivity

- good adhesion

- $\quad$ high thermal stability

- high corrosion resistance

- bondable top-layer

- $\quad$ about zero voltage drop

As the low doped diamond films exhibited semiconducting behaviour, it was necessary to study the metal/diamond contacts to verify if they obey Ohm's law. Most contacts to common semiconductors are depletion contacts which result mainly from the action of surface states. They can show, however, an ohmic behaviour with a linear current-voltage characteristic on degenerated doped semiconductors. In the case of a depletion contact, the contact resistivity varies exponentially with the Schottky barrier height. The ohmic behaviour of the depletion contact can be achieved either when the barrier height is small, so that the charge carriers can easily overcome the barrier (thermionic emission) or when the charge carriers are able to surmount the depletion region by quantum-mechanical tunnelling.

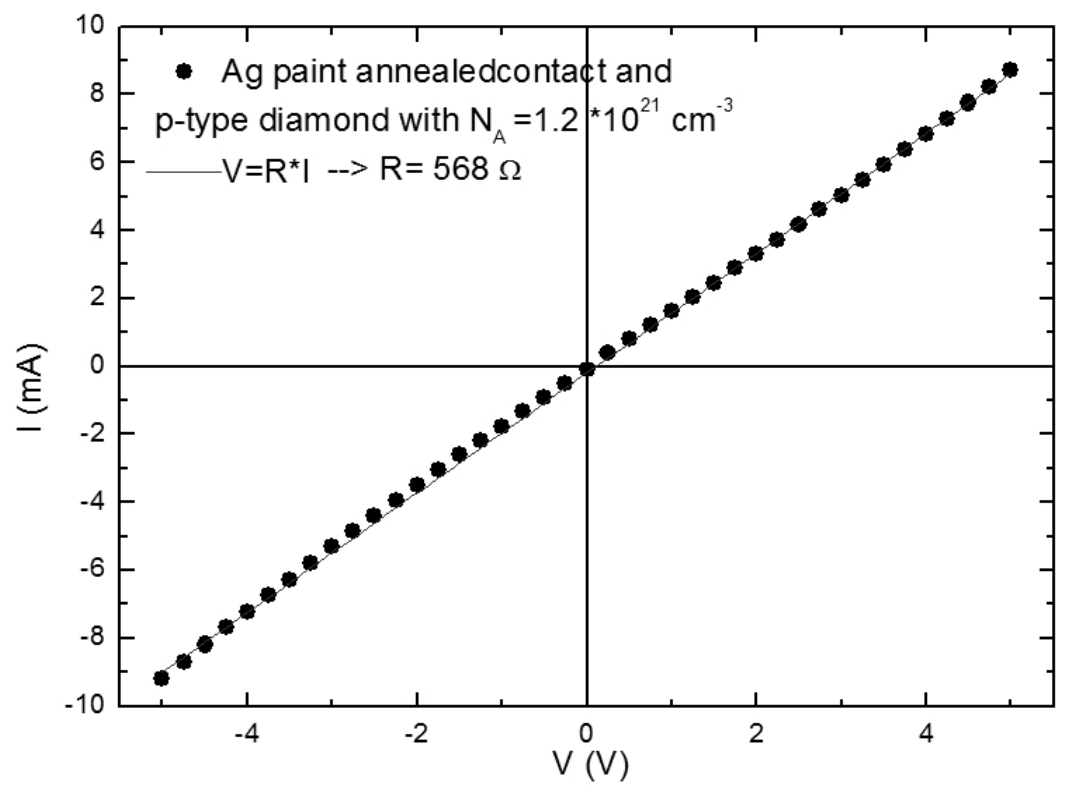

Fig. 4. I-V characteristic of annealed silver paint/ $\mathrm{p}^{+}$-type diamond junction

The depletion layer width of a metal/semiconductor contact is proportional to the square root of the reciprocal doping concentration $\left(\mathrm{W} \sim \mathrm{N}_{\mathrm{A}^{-1 / 2}}\right.$ ) (S.M. Sze, 1981). Consequently, the 
depletion layer width decreases with the increase in doping concentration and the tunnelling probability increases. So a good ohmic contacts are obtained by heavily doping the p-type diamond layer (doping levels much larger than $10^{20} \mathrm{~cm}^{-3}$ ). The resulting layer $\mathrm{p}^{+}$, which is highly doped by $\mathrm{B}$, was metalized by silver paint annealed at $500^{\circ} \mathrm{C}$ for $10 \mathrm{~min}$. The $\mathrm{I}-\mathrm{V}$ characteristic is reported in Fig.4 where is also reported the specific resistance calculated by ohm's law.

\subsection{Shottky contact on intrinsic diamond layer}

The electrical characterization of the metal/intrinsic diamond Schottky junction of the devices was performed at room temperature in a vacuum chamber with a background pressure of $10^{-4}$ mbar by measuring the current-voltage (I-V) characteristics by using a Keithley 6517A pico-ampere meter.

The I-V characteristic was obtained by applying a voltage to the metal contact while the ptype diamond layer is earthing. Fig.5 shows the typical I-V characteristic of the diamond Schottky photodiodes. When the p-type rectifyng contact is reverse biased by connecting the metal to positive terminal, holes are repelled from the interface and the bands are away bent down. The potential barrier for the holes is increase, as is the width of the depletion region. The resulting net current is very low (reverse biased). If instead the metal is connected to the negative terminal, then forward biasing results as the holes are attracted toward the metal interface (forward biased).
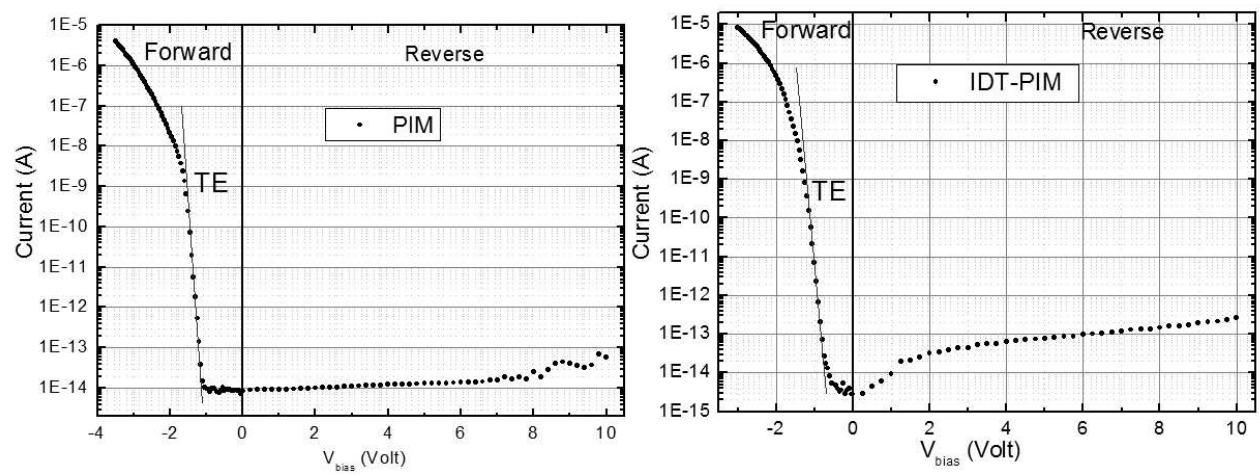

Fig. 5. Typical I-V characteristic of the PIM device

In Fig.5, it's clearly seen the different behaviour of reverse and forward current.

When a negative voltage (forward voltage) is applied on the metal electrode a hole current starts flowing from the p-type diamond, via the nominally intrinsic diamond region, towards the Schottky contact. The rectification behaviour of the both photodiodes is observed with a very high rectification ratio of about $10^{8}$ at $\pm 3 \mathrm{~V}$. For values of $\left|V_{B}\right|<\left|V_{\text {on }}\right|$, where $V_{\text {on }}$ is "turn- on voltage" that in figure is about $-1 \mathrm{~V}$, the forward current is due to generation-recombination effects and leakage superficial current and it's similar at the reverse current. Increasing the forward bias, in the region between approximately $-1 \mathrm{~V}$ and $1.6 \mathrm{~V}$ forward voltage $\left(\mathrm{V}_{\mathrm{F}}\right)$, the current rises exponentially with $\mathrm{V}_{\mathrm{F}}$.

In this region the forward current density $\left(\mathrm{J}_{\mathrm{F}}\right)$ is well described by the thermionic emission (TE) theory. The thermionic emission theory by Bethe is derived from the assumptions that 
the barrier height is much larger than $\mathrm{kT}$, thermal equilibrium is established at the plane that determines emission, and the existence of a net current flow does not affect this equilibrium. Bethe's criterion for the slope of the barrier is that the barrier must decrease by more than $\mathrm{kT}$ over a distance equal to the scattering length. The resulting current flow will depend only on the barrier height and not on the width, and the saturation current is not dependent on the applied bias. Then the current density of majority carriers from the semiconductor over the potential barrier into the metal is expressed as (M. Brezeanu et al., 2007):

$$
\begin{aligned}
& J_{F}=J_{S}\left[\exp \left(\frac{q V_{F}}{n k T}\right)-1\right] \\
& J_{S}=A^{*} T^{2} \exp \left(\frac{-q \phi_{B I}}{k T}\right) \\
& A^{*}=\frac{m_{p}^{*}}{m_{0}} A^{0}
\end{aligned}
$$

where $\mathrm{n}$ is the ideality factor ( $\mathrm{n} \geq 1$ and it informs the experimental I-V characteristic deviates from the behaviour SBD ideal $(\mathrm{n}=1)$ ), $\mathrm{T}$ the absolute temperature (Kelvin), $\mathrm{k}$ the Boltzmann's constant, Js the saturation current density, $A_{0}$ the Richardson's constant $\left(120.173 \mathrm{Acm}^{-2} \mathrm{~K}^{-2}\right), \mathrm{A}^{*}$ the Richardson's effective constant, $\mathrm{m} 0$ and $\mathrm{mp}^{*}$ electron mass and effective mass hole in diamond $\left(\mathrm{m}_{\mathrm{p}}{ }^{*}=0.7 \mathrm{~m}_{0}\right)$ and $\Phi_{\mathrm{BI}}$ the Schottky barrier height. From the exponential fit of the I-V characteristic, it is possible to estimate the saturation current density $\mathrm{J}_{S}$ and the ideality factor $\mathrm{n}$. Substituting the values obtained from the fit in the following equation

$$
\Phi_{B I}=\frac{K_{B} T}{q} \ln \left(\frac{A^{*} T^{2}}{J_{S}}\right)
$$

it's possible estimate the Schottky barrier heigh. The values obtained for IDT-PIM and PIM photodiodes are $1.65 \mathrm{eV}$ and $1.8 \mathrm{eV}$ respectively.

\section{Extreme UV characterization}

The photodiodes have been tested over the extreme UV spectral region from 20 to $120 \mathrm{~nm}$, using $\mathrm{He}$ and $\mathrm{He} \mathrm{Ne} \mathrm{DC}$ gas discharge as radiation sources and a toroidal grating vacuum monochromator (Jobin Yvon model LHT 30) with a $5 \AA$ wavelength resolution. The dimension of the optical aperture is $0.25 \times 6.00 \mathrm{~mm}^{2}$; a manual shutter is used to switch on and off the UV radiation. The experimental apparatus of UV characterization is reported in the following picture.

The photoresponse measurements have been performed in a vacuum chamber, at a pressure of 0.03 mbar. By using a three (X-Y-Z) dimension mechanical stage powered by stepper motors, it is possible to locate the photodetector in front of the beam light and to compare its response with that of a calibrated NIST silicon photodiode (http://www.ird-inc.com) placed in the same position, which measures the absolute photon flux. A raster scansion of the beam light was performed on the detector surface so to position the photodetectors where their response has a maximum (see Fig.6(b)). 

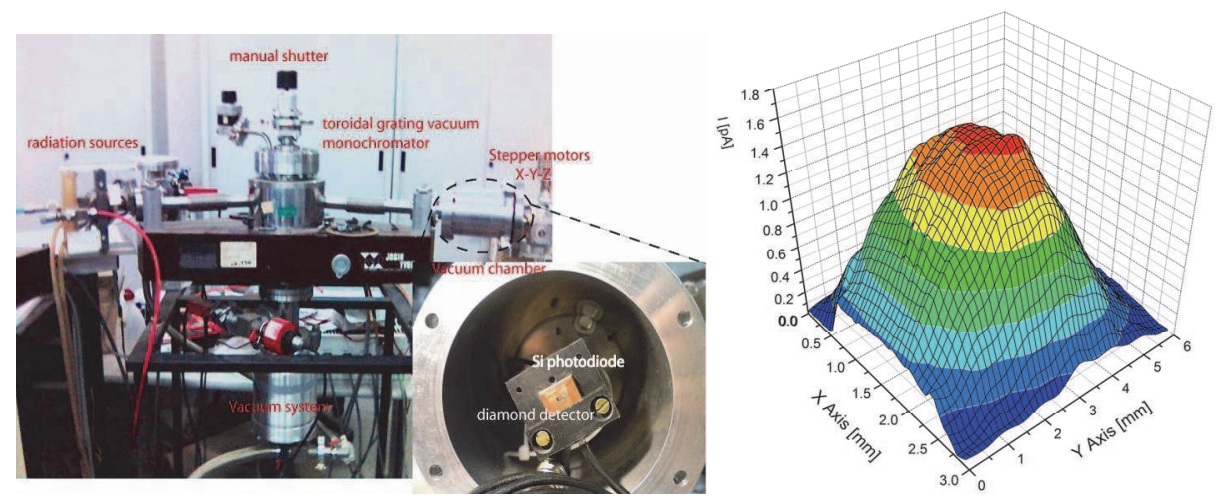

Fig. 6. a) Extreme UV characterization system, b) Raster scansion of the beam light

A hole, $2 \mathrm{~mm}$ in diameter, is used to collimate the radiation on the sensitive area of the detectors and to obtain the same illuminated area on the silicon photodiode. The photocurrent is measured by an electrometer (Keithley 6517A), using the internal voltage source.

Because of different geometry adopted by the two devices, they are measured differently. The PIM detector is encapsulated in a copper/vetronite shielded housing with a $2 \mathrm{~mm}$ pinhole. In such housing, the $\mathrm{Al}$ contact is grounded and the photocurrent is measured from p-type diamond so that the signal is not affected by the eventual presence of secondary electron emission current from the illuminated contact.
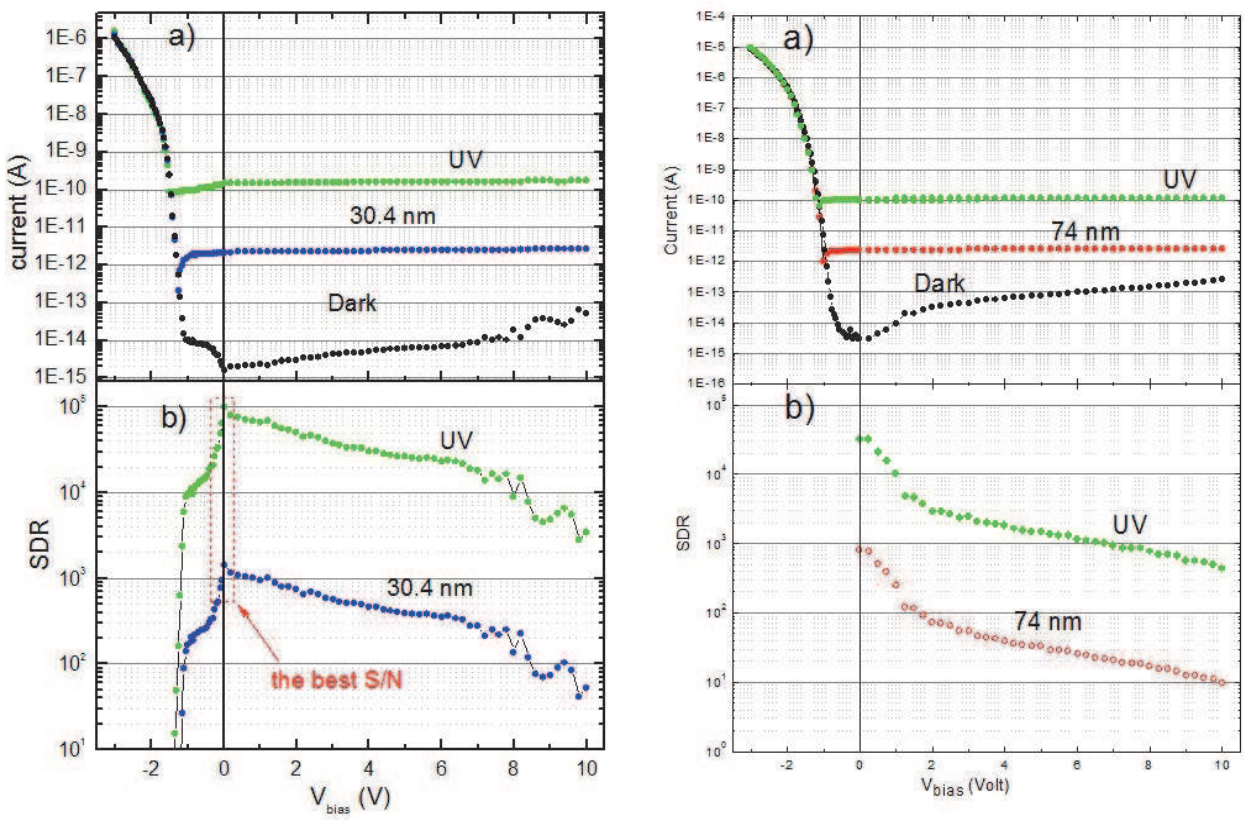

Fig. 7. a) I-V characteristic in dark and in light of PIM detectors and b) signal to dark current ratio (SDR) 
The IDT-PIM is simply mounted in an sample holder for UV measurements with the same 2 $\mathrm{mm}$ pinhole. In this case, the measured photocurrent of IDT-PIM detector can contain both photoemission current and photoconductive current. The photoemission contribution contains electron emission arising from $\mathrm{Al}$ fingers and from $\mathrm{p}$-type diamond exposed to the UV irradiation. The typical current - voltage (I-V) characteristics in dark current and under irradiation have been measured at room temperature of two detectors are shown in Fig. 7.

The devices operate in the reverse bias mode because when operating in the forward bias mode, the photocurrent is masked by the dark current. The dark current is very low $(<0.1$ $\mathrm{pA}$ ) below about $+10 \mathrm{~V}$, as expected for a metal/diamond rectifying contact.

The photocurrent vs. applied voltage is also reported in the same figure when the device is exposed to UV radiation and $30.4 \mathrm{~nm}$ (He lines) and $73 \mathrm{~nm}$ (Ne line). The device shows a photocurrent response even at zero voltage bias, exploiting the internal junction electric field. The photocurrent is almost constant with increasing positive voltage, while the dark current increases by about two orders of magnitude. Remarkably, thus, the best signal-todark current (SDR) ratio (see Fig.7 (b)) is obtained at zero bias voltage, so that in the following, the devices have been operated with no external bias voltage applied.

\subsection{Temporal response}

Temporal response measurements upon exposure to UV radiation have been performed according to the following procedure: at first the dark current value was recorded for several seconds keeping the light shutter closed, until the steady state value had been reached; then the shutter was opened and the photocurrent was measured. Finally, the shutter was closed again until the dark current reached the initial value, before starting a new measurement run. The detectors time response, upon exposure to UV radiation, have been measured by opening and closing a manual shutter during the acquisition. The temporal response of the tested devices is reported in Fig. 8 (a) under UV illumination of the $\mathrm{He}-\mathrm{Ne}$ DC gas discharge radiation source.

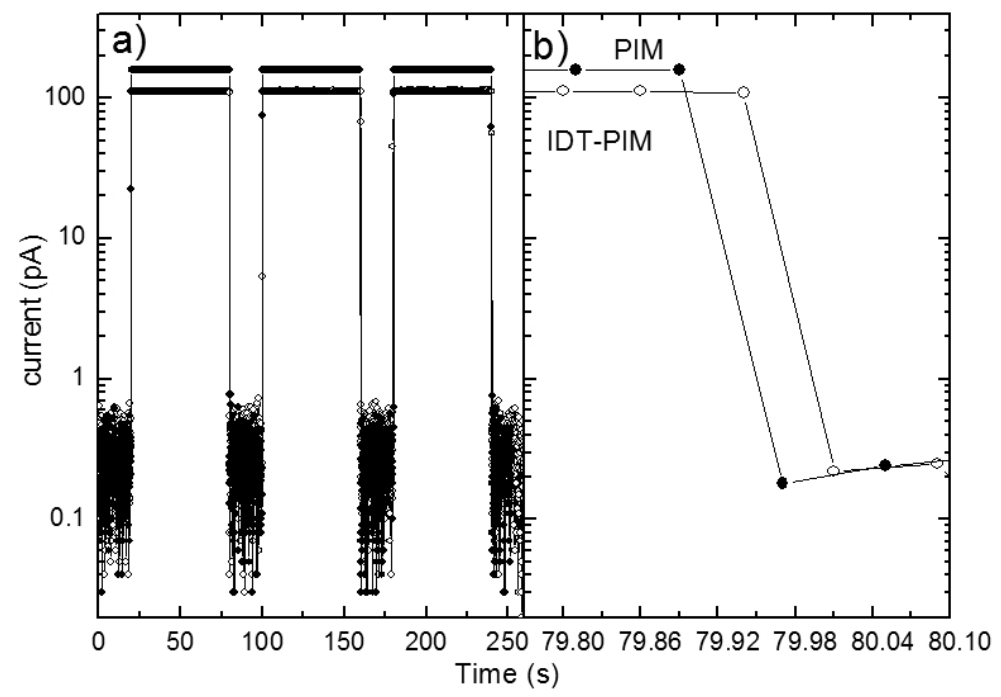

Fig. 8. a) Temporal responses under illumination of He-Ne DC gas discharge radiation source. b) The magnification of fall time of the both devices. 
The response is reproducible and no undesired effects such as persistent photocurrent and priming or memory effects, which are often observed in diamond UV detectors (C. E. Nebel et al., 2000, A. De Sio et al., 2005, M. Liao et al., 2008), are observed. negligible. However, it is obtained only after the very first irradiation: the device, just mounted, reaches the described performance only after a pre-irradiation time of few minutes Fig.8 (b) shows rise and fall times of the signal of about $60 \mathrm{~ms}$, which corresponds to the acquisition rate of the used electronic chain.

\subsection{Linearity}

A useful detector is expected to exhibit linear response with photon flux, i.e. a constant responsivity up to a saturation point where space charge effects prevail and no more electron-hole pairs can be collected under illumination. The calibration of linear detectors and related electronics is much simpler. The linearity of the photodetectors have been investigated varying the current intensity of the plasma. The photocurrent $\left(\mathrm{I}_{\mathrm{ph}}\right)$ measured vs. the incident optical power $(\mathrm{P})$, under irradiation of He-Ne gas discharge radiation is shown in Fig.9. We used a power law: $I_{p h}=A+B \bullet P C$ to fit the data. Here $A$ is the offset corresponding to the dark current, $B$ is the photosensitivity (provided $C=1$ ) and $C$ is a linearity coefficient. The graph shows the measured data as well as the fitting function. In this spectral region, both photodetectors shows remarkably good linearity, $\mathrm{C}$ being 1 in all cases within the error.
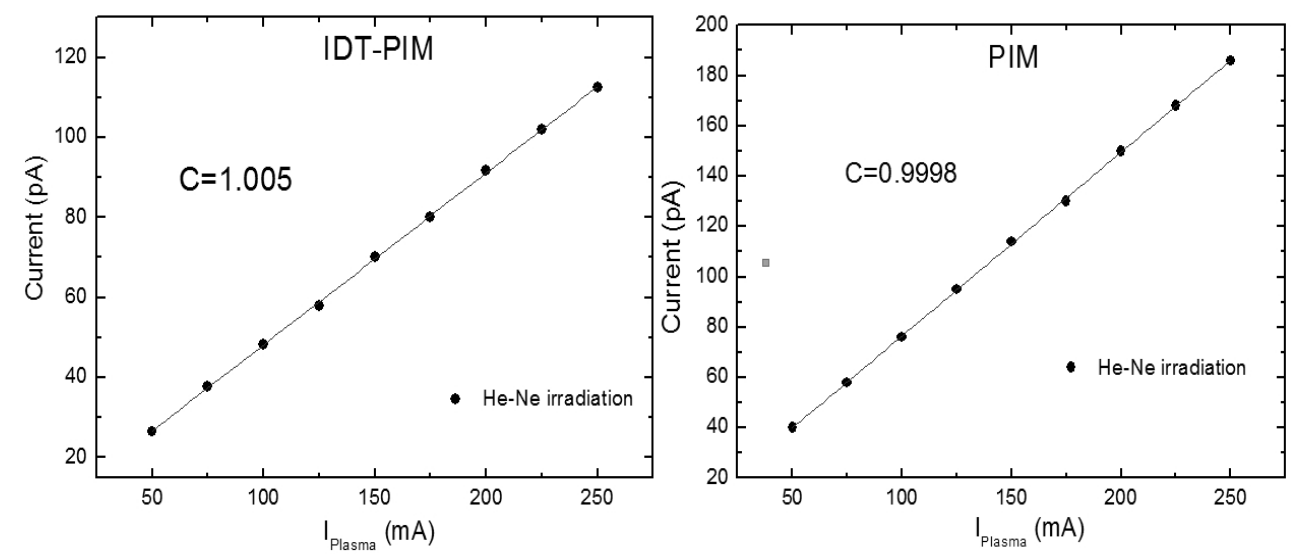

Fig. 9. Linearity of IDT-PIM and PIM photodiodes.

\subsection{Extreme UV spectroscopy}

The normalized emission spectra of a DC discharge He and He-Ne lamp measured in unbiased mode by the detectors are reported in Fig.10.

All spectral lines are clearly resolved and observed with a good signal to noise ratio, demonstrating the high photodetection capabilities of the CVD single crystal diamond grown in the extreme UV spectral region. All spectral lines are classified by the NIST Atomic Spectra Database Lines Form from the following website:

http:// physics.nist.gov/PhysRefData/ASD/lines_form.html. 

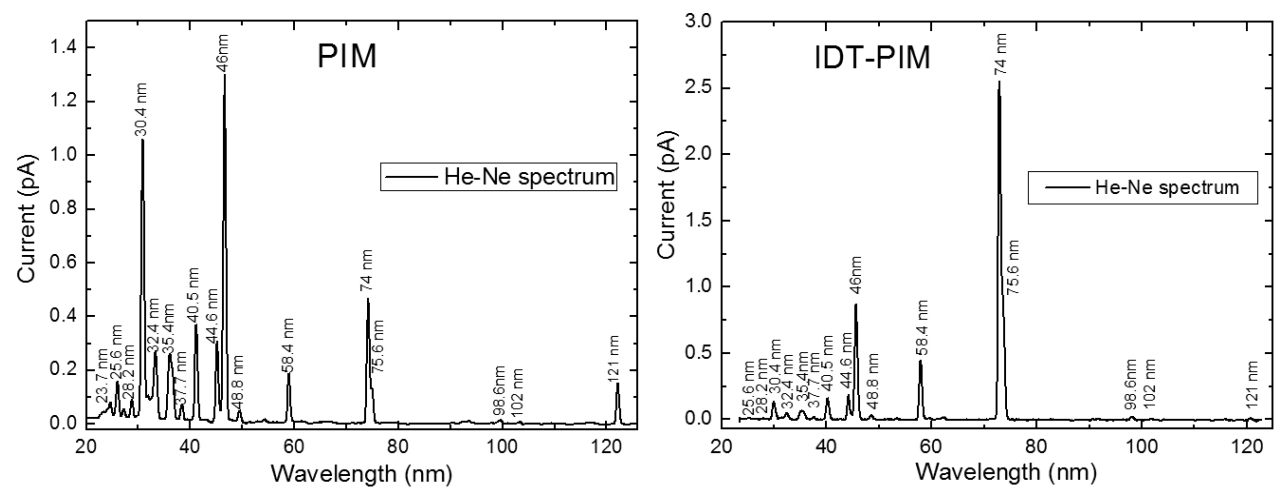

Fig. 10. He-Ne emission spectrum measured by the two devices.

In particular, the low intensity lines of the He-Ne spectrum in the wavelength range 20-30 $\mathrm{nm}$ are easily resolved by PIM detector

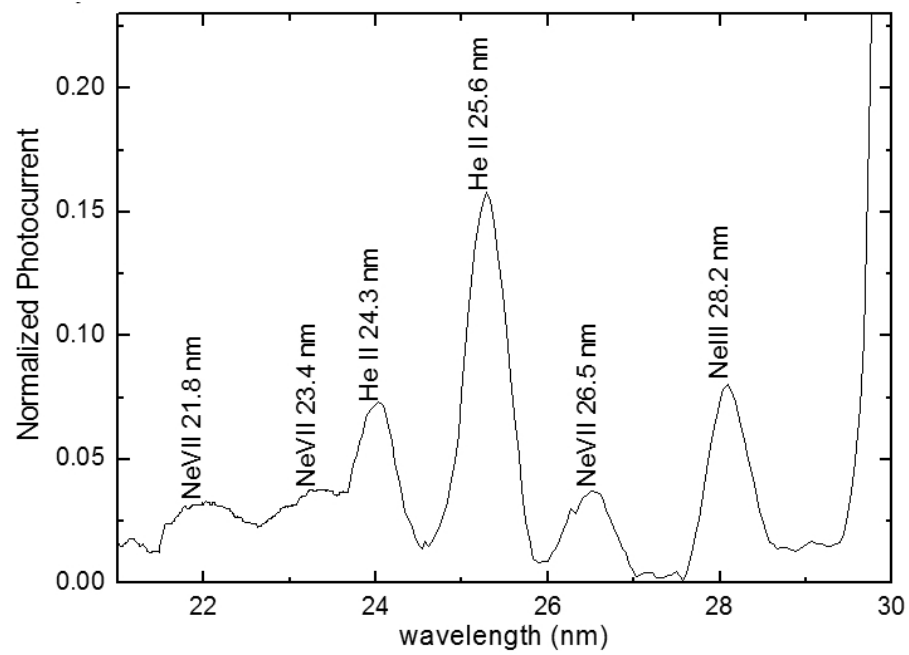

Fig. 11. He-Ne spectrum measured by PIM detector in the range $20-30 \mathrm{~nm}$

\subsection{Responsivity and external quantum efficiency}

The absolute spectral response of the PIM detectors is measured by comparison with a calibrated photodiode exposed to the same source on the same optical area of about $1 \mathrm{~mm} 2$. The spectral responsivity, expressed in amperes per watt $(\mathrm{A} / \mathrm{W})$, is defined as the photocurrent per unit incident optical power and can be evaluated from the relationship $R_{d}$ $=R_{\mathrm{Si}} \mathrm{I}_{\mathrm{d}} / \mathrm{I}_{\mathrm{Si}}$ where $\mathrm{R}_{\mathrm{Si}}$ is the responsivity of the calibrated silicon photodiode at a given wavelength, $\mathrm{I}_{\mathrm{Si}}$ and $\mathrm{I}_{\mathrm{d}}$ are the photocurrents measured by the silicon photodiode and the diamond detector, respectively.

The responsivities of both photodiodes are reported in Fig.12. The responsivity of the PIM device decreases monotonically as the wavelength increases until about $80 \mathrm{~nm}$ while at 120 
$\mathrm{nm}$ an increased value is observed. At $98 \mathrm{~nm}$ the signal is below the noise level so that only an upper limit can be provided. However, the presence of a minimum in the responsivity around $100 \mathrm{~nm}$ can be clearly deduced from Fig.12.

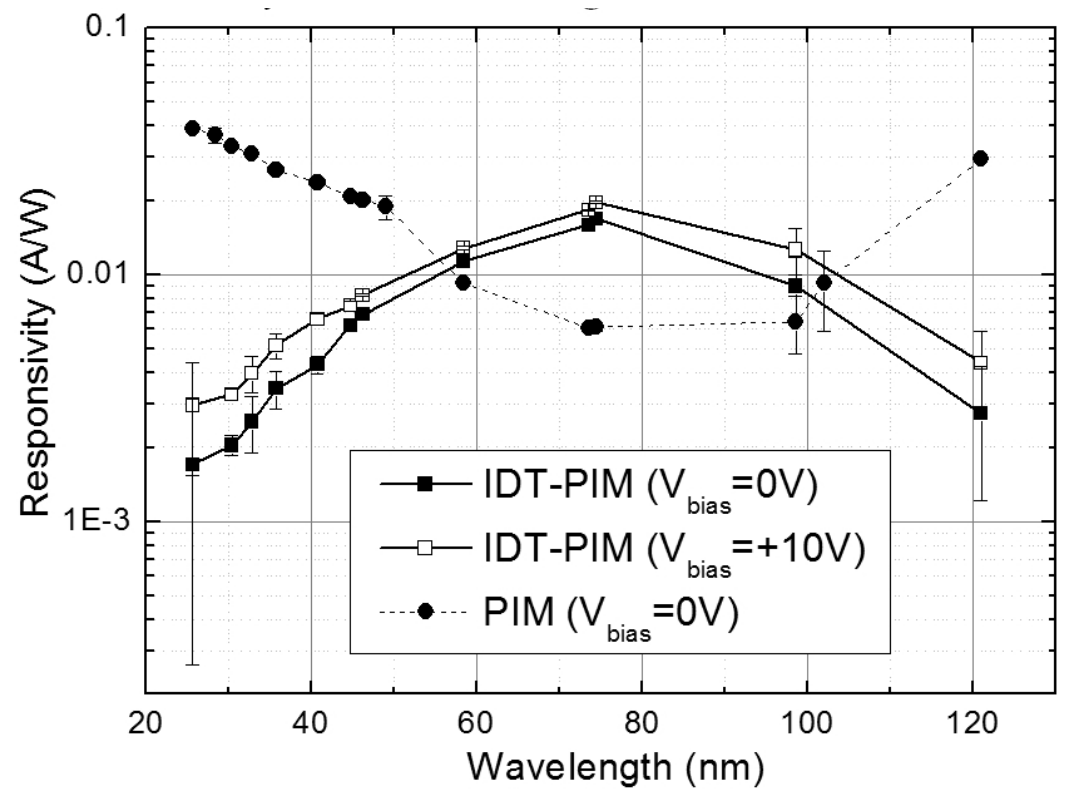

Fig. 12. Responsivity of the both devices.

The responsivity of the IDT-PIM detector is much lower than that of the PIM detector at short wavelength (below $50 \mathrm{~nm}$ ) showing a maximum at about $73 \mathrm{~nm}$. The increased sensitivity of the IDT-PIM device at intermediate wavelength could be probably ascribed to the contribution of photoemission current as already reported in the literature (T. Saito et al, 2006). For both the devices the absolute responsivity measured at around $50 \mathrm{~nm}$ is comparable to the best results reported in the literature for diamond based EUV detectors (A. BenMoussa et al., 2006).

The External Quantum Efficiency (EQE) spectrum, estimated by: EQE $=1240 \bullet \operatorname{Rd} / \lambda[\mathrm{nm}]$, is reported in Fig.7 for the PIM devices.

As mentioned above, the photocurrent measured by IDT-PIM detector includes the contains both photoconductive current and photoemission current, arising from secondary electron escape from Al fingers, which also depends on the wavelength (J. Ristein et al, 2005, W. Pong et al., 1970). On the contrary, in the encapsulated PIM device the illuminated contact is grounded and the current flowing from the boron doped layer is not affected by secondary electrons contribution. Moreover, the more homogeneous electric field configuration of the PIM device allows a simple analysis of the detection process.

In order to investigate the effect of the metallic Schottky contact upon the detection performance of the PIM devices, different semitransparent metals (thickness < 10nm) have been thermally evaporated on the oxidized surface of single crystal CVD intrinsic diamond layers. 


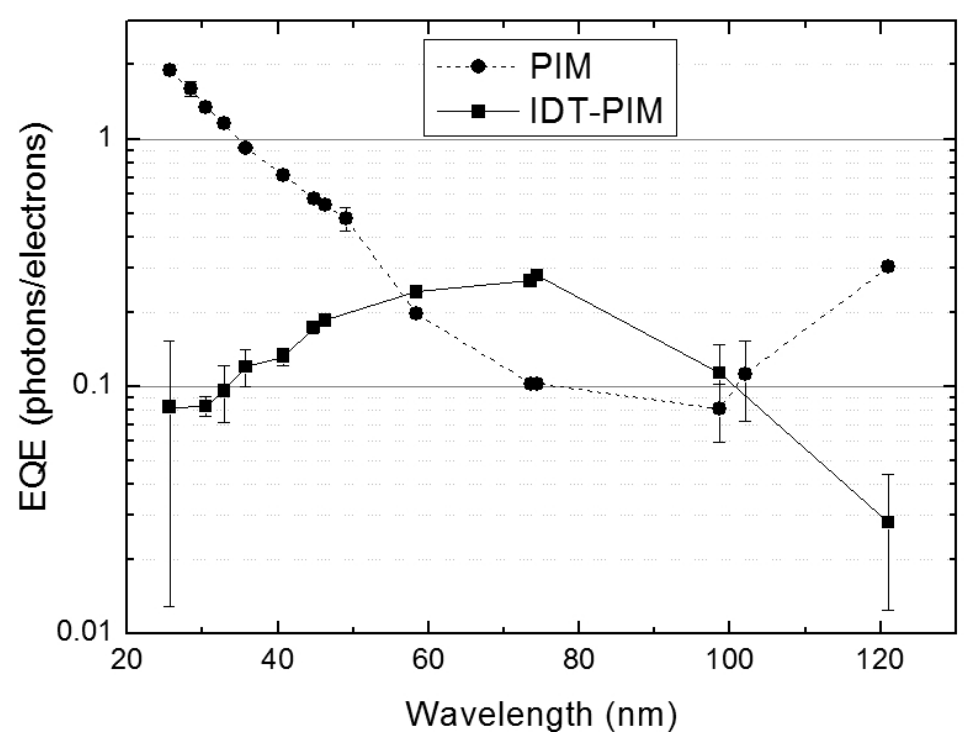

Fig. 13. External quantum efficiency EQE of the two photodiodes between 20 and $120 \mathrm{~nm}$.

The absolute spectra responsivity curves versus different meal contacts of the devices are shown in Fig.14. All the devices have a maximum of the responsivity at lower wavelengths and a sharp cutting edge for longer wavelengths while at around $120 \mathrm{~nm}$ an increased value is observed. The lowest responsivity, between $50 \div 100 \mathrm{~nm}$, has been measured for the device having $\mathrm{Cr}$ as an electrode. The device having Ag and Pt contacts shows rather similar trend of the responsivity, whereas $\mathrm{Al}$ contact shows the best results in the UV performances.

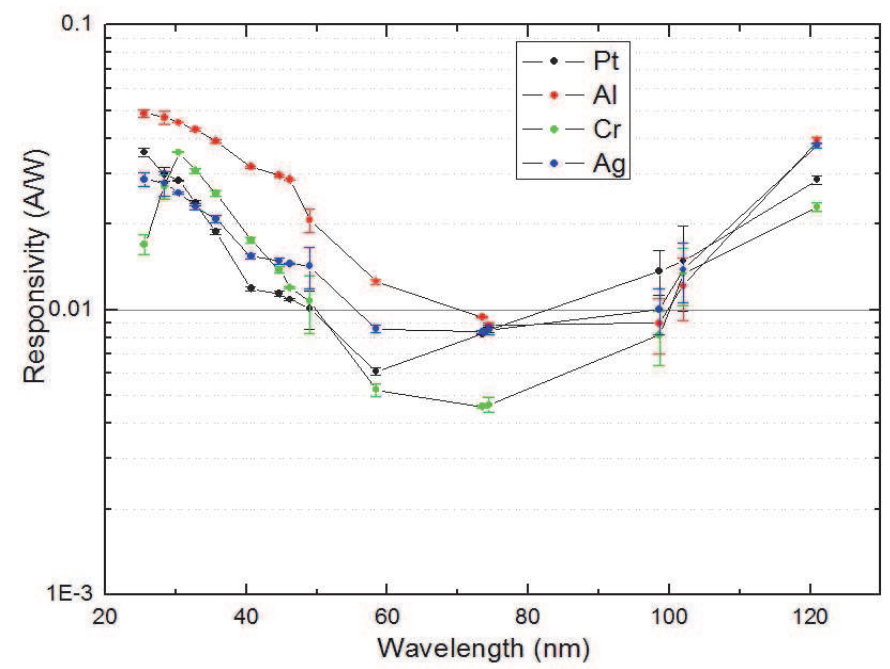

Fig. 14. External quantum efficiency EQE of the PIM devices between 20 and $120 \mathrm{~nm}$ as a function of the type of the metallic contact. 


\subsection{UV/visible rejection ratio}

The photoconductive response was tested over a wide spectral range, extending from the extreme UV (EUV) up to the visible. The $210-500 \mathrm{~nm}$ range was investigated using an Optical Parametric Oscillator (OPO) 5 ns pulsed laser (Opolette laser by Opotek). The laser beam was scattered by an optical diffuser in order to prevent signal saturation of the electronic chain and the diamond detector was placed $10 \mathrm{~cm}$ away from the diffuser. A 500 $\mathrm{MHz}$ Le Croy WaveRunner 6050 digital oscilloscope was used to acquire the output signal.
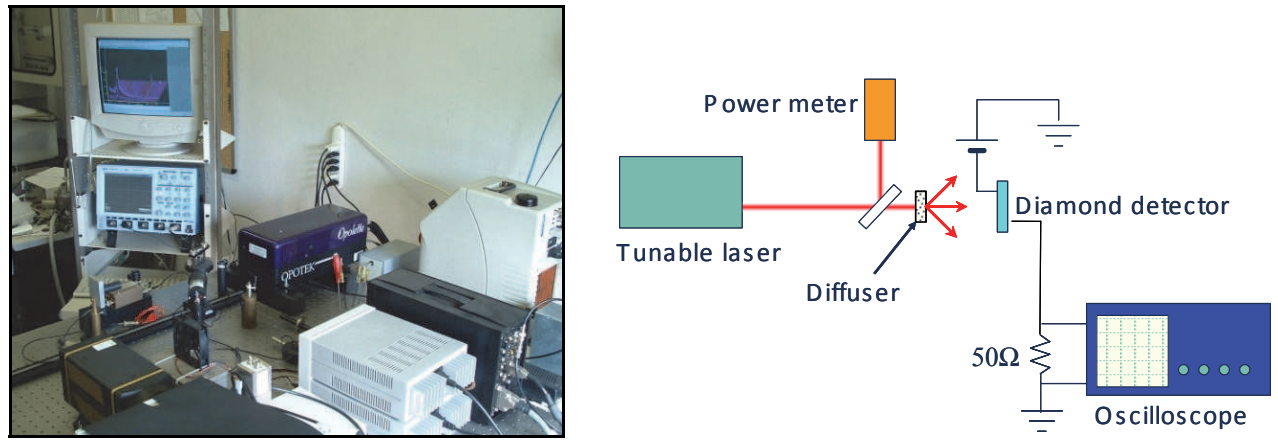

Fig. 15. Optical Parametric Oscillator and experimental set up.

Two different connection configurations were used:

i. Direct recording of the detector output by the digital oscilloscope

ii. Integrated measurement by an Ortec142A charge preamplifier.

The signal provided by a pyroelectric power meter was used to normalize the diamond detector output, in order to take into account the wavelength dependence of laser pulse amplitude and the intrinsic fluctuations of the beam intensity.

The visible-blind properties of the photodetectors were tested by measuring the photoresponse at different wavelengths in the $210-500 \mathrm{~nm}$ range.

In Fig.16 (a) the device responsivity of the PIM detector is reported as a function of the incident laser radiation wavelength, normalized to the pyroelectric power meter signal. A 3 orders of magnitude variation was measured when moving across the band gap wavelength of $225 \mathrm{~nm}$. Such a drop increases up to 5 orders of magnitude when the UV to visible rejection ratio is considered. It should be stressed that a very stable and reproducible response was observed in the whole energy range and irradiation memory or pumping effects were not observed.

In addition, a linear increase in the photoresponse as a function of calculated radiation intensity was observed measuring the output signal at decreasing device distances from the optical diffuser.

The time response at $220 \mathrm{~nm}$ of the investigated PIM detector is reported in Fig. 16 (b). As clearly seen in the Fig.16 (b), the device response to a laser pulse at $220 \mathrm{~nm}$, measured through a bias Tiee and recording by the digital oscilloscope (Le Croy $500 \mathrm{MHz}$ ), shows an exponential decay time constant of about 100ns. The reason of this trend of output response is due to electrical circuit of the device. In fact, an RC circuit, the value of the time constant is equal to the product of the circuit resistance and the circuit capacitance. Therefore, taking into account the depletion capacitance measured by $\mathrm{C}-\mathrm{V}$ curves of about $100 \mathrm{pF}$ and the resistance of p-type diamond film $\sim 1 \mathrm{k} \Omega$, the time constant result to be $\tau=100 \mathrm{~ns}$. 

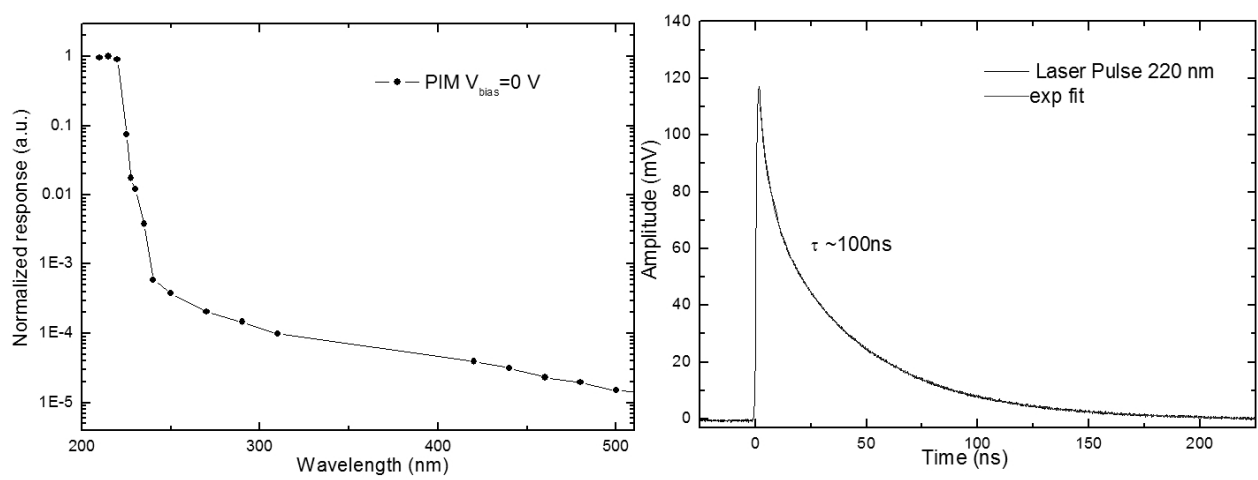

Fig. 16. a)Normalized responsivity of PIM device as a function of the incident laser radiation wavelength. b) The device response to laser pulses directly obtained by the digital oscilloscope.

The visible-blind properties of the IDT-PIM device were also tested by measuring the photoresponse at different wavelengths in the $210-500 \mathrm{~nm}$ range. In this region, the spectral response shows a visible/UV rejection ratio of about $4 / 5$ orders of magnitude, as clearly seen in Fig.17(a) .Moreover, the time response at $220 \mathrm{~nm}$ of the investigated detector is reported in Fig.17 (b). The Fig.17(b) shows the device response to a laser pulse at $220 \mathrm{~nm}$, which have a full width at half maximum (FWHM) of about $25 \mathrm{~ns}$, and the time response is faster than that of PIM detector. In fact, in this case, the parallel capacitance of the photodiode is very low, about $15 \mathrm{pF}$.

Interdigitated structure, therefore, can be optimized in order to build a ultrafast XUV detector, for time resolution.
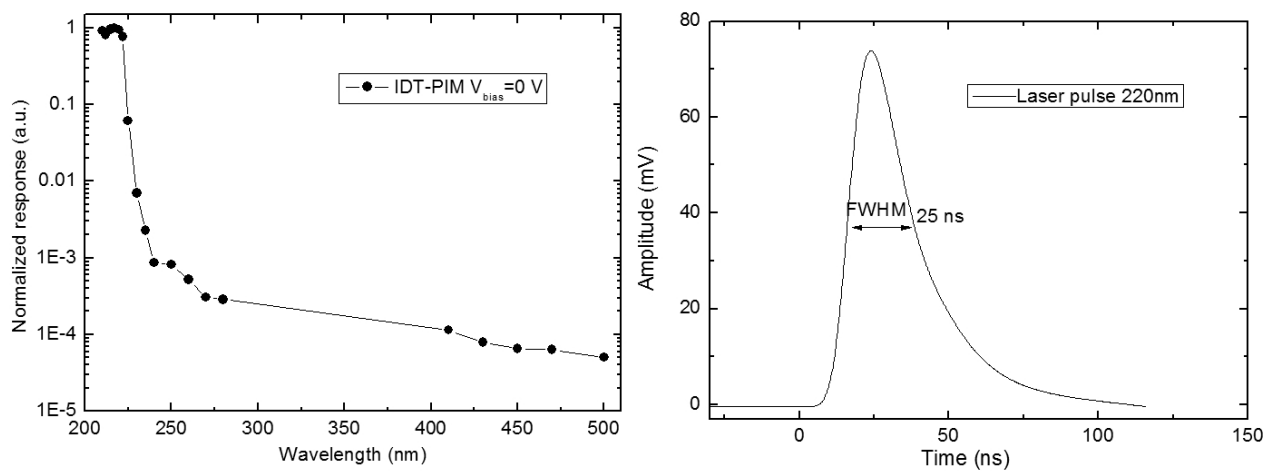

Fig. 17. a) Normalized responsivity of IDT- PIM device as a function of the incident laser radiation wavelength. b) The device response to laser pulses directly obtained by the digital oscilloscope.

\section{Conclusion}

Two detectors were fabricated at the University of Rome "Tor Vergata" with a structure that acts as a metal/intrinsic/p-doped diamond photovoltaic Schottky diode. The two detectors 
operate in different configurations: one in transverse geometry and the other one in planar configuration.

We have measured the electrical characteristics and tested the performance under continuous vacuum UV photon irradiation of the two devices. A general result of our experiments is that diamond detectors are very sensitive devices showing show a very low dark current and very good signal-to-noise ratio. The responses are reproducible and undesired effects such as persistent photocurrent, priming or memory effects are negligible for both devices. The response time could is very fast and it is much lower than the acquisition rate of the used electronic chain $(\sim 60 \mathrm{~ms})$. These results indicate the high quality of our CVD diamond grown for UV applications.

The responsivity and the EQE of the two devices show an opposite behaviour as a function of the radiation wavelengths due to the different operative configurations. In particular the PIM detector is more efficient at lower wavelengths and present a drop of sensitivity at approximately $100 \mathrm{~nm}$. The IDT-PIM is less efficient at low wavelength and has a maximum efficiency at about $74 \mathrm{~nm}$.

The visible-blind properties of the photodetector were also tested by measuring the photoresponse at different wavelengths in the 210-500 $\mathrm{nm}$ range. A 3/4 orders of magnitude variation was measured by diamond based detectors when moving across the band gap wavelength of $225 \mathrm{~nm}$. Moreover, the spectral response shows a visible/UV rejection ratio of about 5 orders of magnitude for both photodiodes. Finally, the device response to laser pulses at $220 \mathrm{~nm}$ is different in two cases due to the different electrical circuit of the two devices. In particular, the time response of IDT-PIM detector is faster than that of PIM detector.

\section{Acknowledgment}

The devices studied in this chapter were developed by the group of Rome University "Tor Vergata" composed by Prof. Marco Marinelli, Prof. Enrico Milani, Dr Gianluca VeronaRinati, Dr Giuseppe Prestopino and myself. They have made possible the writing of this chapter.

Moreover, I'd like to thank the staff of "O. M. Corbino" Institute of Acoustics (IDAC) of CNR who give me the possible to perform the photolithography techniques used to realize the devices developed in this chapter.

\section{References}

J. E. Field, Properties of Diamond, Academic Press, London, (1979).

J.Prins, Applications of diamond films in electronics in "The Physics of Diamond", A. Paoletti and A. Tucciarone (editors), IOS Press, Amsterdam, (1997).

J.F. Hochedez, J. Alvarez, F.D. Auret, P. Bergonzo, M.-C. Castex, A. Deneuville, J.M. Defise, B. Fleck, P. Gibart, S.A. Goodman, O. Hainaut, J.-P. Kleider, P. Lemaire, J. Manca, E. Monroy, E. Munoz, P. Muret, M. Nesladek, F. Omnes, E. Pace, J.L. Pau, V. Ralchenko, J. Roggen, U. Schuhle, C. Van Hoof, Diamond Relat. Mater. 11 (2002), 427.

A. Balducci, M. Marinelli, E. Milani, M.E. Morgada, A. Tucciarone, G. Verona-Rinati, M. Angelone, M. Pillon, Appl. Phys. Lett. 86 (2005), 193509.

T. Teraji, S. Yoshizaki, H. Wada, M. Hamada, T. Ito , Diamond Relat. Mater. 13 (2004), 858. 
V.I. Polyakov, A.I. Rukovishnikov, N.M. Rossukanyi, A.I. Krikunov, V.G. Ralchenko, A.A. Smolin, V.I. Konov, V.P. Varnin, I.G. Teremetskaya, Diamond Relat. Mater. 7 (1998), 821.

L. Thaiyotin, E. Ratanaudompisut, T. Phetchakul, S. Cheirsirikul, S. Supadech, Diamond Relat Mater 11 (2002), 442.

R. D. McKeag and R. B. Jackman, Diamond Relat. Mater. 7 (1998), 513.

L. Barberini, S. Cadeddu, and M. Caria, Nucl. Instrum. Methods 460 (2001), 127.

E. Pace, A. Vinattieri, A. Pini, F. Bogani, M. Santoro, G. Messina, S. Santangelo, Y. Sato, Phys. Status Solidi, A Appl. Res. 181 (2000), 91.

S. Almaviva, Marco Marinelli, E. Milani, G. Prestopino, A. Tucciarone, C. Verona, G. Verona-Rinati, M. Angelone, M. Pillon, Diamond Relat. Mater. 18 (2009), 101.

S. Almaviva, Marco Marinelli, E. Milani, G. Prestopino, A. Tucciarone, C. Verona, G. Verona-Rinati, M. Angelone, M. Pillon, I. Dolbnya, K. Sawhney and N. Tartoni, J. Appl. Phys. 107014511 (2010).

S. Almaviva, Marco Marinelli, E. Milani, G. Prestopino, A. Tucciarone, C. Verona, G. Verona-Rinati, M. Angelone, M. Pillon Diamond and Related Materials, v 19, n 1, p 7882, January 2010.

D. Palik., Handbook of Optical Constants of Solids II, Academic Press, New York (1991).

M. Werner, Semicond. Sci. Technol. 18 (2003) S41-S46.

S.M. Sze, Physics of Semiconductor Devices, John Wiley and Sons (WIE) (1981)

M. Brezeanu, T. Butler, N. Rupesinghe, S. J. Rashid, M. Avram, G. A. J Amaratunga, F. Udrea, M. Dixon, D. Twitchen, A. Garraway, D. Chamund, and P. Taylor, IEEE Proc.: Circuits Devices Syst. 1, 380 (2007).

website: http://www.ird-inc.com.

C. E. Nebel, A. Waltenspiel, M. Stutzmann, M. Paul, and L. Schäfer, Diamond Relat. Mater. 9, (2000), 404.

A. De Sio, E. Pace, Nucl. Instr. Methods A 552 (2005), 203

M. Liao, Y. Koide, J. Alvarez, M. Imura, J.P., Physical Review B 78 (2008), 045112.

T. Saito, K. Hayashi, H. Ishihara and I. Saito, Metrologia 43, (2006), S51.

A. BenMoussa, A. Theissen, F. Scholze, J.F. Hochedez, U. Schuhle, W. Schmutz, K. Haenen, Y. Stockman, A. Soltani, D. McMullin, R.E. Vest, U. Kroth, C. Laubis, M. Richter, V. Mortet, S. Gissot, V. Delouille, M. Dominique, S. Koller, J.P. Halain, Z. Remes, R. Petersen, M. D'Olieslaeger, J.M. Defise , Nucl. Instr. Methods A 568 (2006), 398.

J. Ristein, W. Stein, L. Ley, Diamond and Relat. Mat. 7, 626 (1998).

W. Pong, R. Sumida, G. Moore, J. Appl. Phys 41, 1869 (1970). 


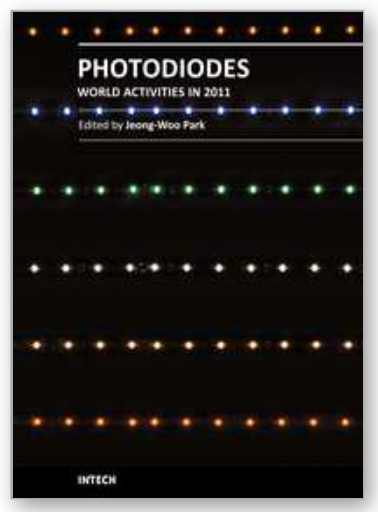

\author{
Photodiodes - World Activities in 2011 \\ Edited by Prof. Jeong Woo Park
}

ISBN 978-953-307-530-3

Hard cover, 400 pages

Publisher InTech

Published online 29, July, 2011

Published in print edition July, 2011

Photodiodes or photodetectors are in one boat with our human race. Efforts of people in related fields are contained in this book. This book would be valuable to those who want to obtain knowledge and inspiration in the related area.

\title{
How to reference
}

In order to correctly reference this scholarly work, feel free to copy and paste the following:

Claudio Verona (2011). Single Crystal Diamond Schottky Photodiode, Photodiodes - World Activities in 2011, Prof. Jeong Woo Park (Ed.), ISBN: 978-953-307-530-3, InTech, Available from:

http://www.intechopen.com/books/photodiodes-world-activities-in-2011/single-crystal-diamond-schottkyphotodiode

\section{INTECH}

open science | open minds

\section{InTech Europe}

University Campus STeP Ri

Slavka Krautzeka 83/A

51000 Rijeka, Croatia

Phone: +385 (51) 770447

Fax: +385 (51) 686166

www.intechopen.com

\section{InTech China}

Unit 405, Office Block, Hotel Equatorial Shanghai

No.65, Yan An Road (West), Shanghai, 200040, China

中国上海市延安西路65号上海国际贵都大饭店办公楼 405 单元

Phone: +86-21-62489820

Fax: $+86-21-62489821$ 
(C) 2011 The Author(s). Licensee IntechOpen. This chapter is distributed under the terms of the Creative Commons Attribution-NonCommercialShareAlike-3.0 License, which permits use, distribution and reproduction for non-commercial purposes, provided the original is properly cited and derivative works building on this content are distributed under the same license. 